

\title{
Non-perturbative F-terms across lines of BPS stability
}

\author{
I. García-Etxebarria, ${ }^{a b}$ F. Marchesano ${ }^{a}$ and A.M. Uranga ${ }^{a b}$ \\ ${ }^{a} \mathrm{PH}-\mathrm{TH}$ Division, CERN, \\ CH-1211 Geneva 23, Switzerland \\ ${ }^{b}$ Instituto de Física Teórica UAM/CSIC, \\ Universidad Autónoma de Madrid C-XVI, \\ Cantoblanco, 28049 Madrid, Spain \\ E-mail: inaki@cern.ch, marchesa@cern.ch, Angel.Uranga@cern.ch
}

\begin{abstract}
We consider non-perturbative terms in the 4d effective action due to BPS D-brane instantons, and study their continuity properties in moduli space as instantons cross lines of BPS stability, potentially becoming non-BPS. We argue that BPS instantons contributing to the superpotential cannot become non-BPS anywhere in moduli space, since they cannot account for the required four goldstino fermion zero modes. At most they can reach lines of threshold stability, where they split into mutually BPS multi-instantons, as already discussed in the literature. On the other hand, instantons with additional fermion zero modes, contributing to multi-fermion F-terms, can indeed cross genuine lines of marginal stability, beyond which they lead to non-BPS systems. The non-BPS instanton generates an operator which is a D-term locally in moduli space, but not globally. This is due to a cohomological obstruction localized on the BPS locus, where the D-term must be written as an F-term, thus ensuring the continuity of the $4 \mathrm{~d}$ contribution to the effective action. We also point out an interesting relation between lifting of fermion zero modes on instantons and $4 \mathrm{~d}$ supersymmetry breaking.
\end{abstract}

KEYwords: Brane Dynamics in Gauge Theories, Nonperturbative Effects, D-branes. 


\section{Contents}

1. Introduction 1

2. Review of higher F-terms

3. The isolated $U(1)$ instanton 5

3.1 The setup

3.2 On the BPS locus 6

3.3 Away from the BPS locus

3.4 The near BPS regime 9

3.5 The global picture 10

3.6 Application to lines of marginal stability 10

4. The $N_{f}=N_{c}$ SQCD instanton from branes 12

4.1 The setup 12

4.2 At the BPS locus 13

4.3 The near-BPS regime 14

4.4 The deep non-BPS regime 16

5. Lifting of fermion zero modes and 4d supersymmetry breaking 17

5.1 Gauge theories with non-perturbative superpotential 17

5.2 Closed string fluxes 18

5.3 Effects of additional instantons 20

6. Conclusions 21

A. Some useful geometries

\section{Introduction}

An important aspect of the dynamics of string theory compactifications is the study of non-perturbative effects, arising from euclidean brane instantons (see e.g. [1- [1]). Indeed, recent applications of D-brane instantons in type II compactifications (or F/M-theory duals) include, among others, mechanisms of moduli stabilization [5-7], generation of perturbatively forbidden couplings [8, 9] (see also [10-12]), generation of gauge field theory superpotentials [13-17], and realization of supersymmetry breaking [18-20]. Other aspects of D-brane instantons have been recently discussed in e.g. 21-26].

Some of these applications, like moduli stabilization, involve the use of the instantoninduced $4 \mathrm{~d}$ operators throughout moduli space. The computation of such global expression 
for non-perturbative effects would seem feasible for $4 \mathrm{~d}$ F-terms (e.g. superpotentials) which are holomorphic on all moduli and thus nicely behaved. However this question is subtler than it seems, since the non-perturbative contributions to a $4 \mathrm{~d}$ F-terms depends in principle on the spectrum of BPS instantons at each point in moduli space, and this spectrum can jump discontinuously across lines of BPS stability (see e.g. [27]), which are generically real codimension one loci in moduli space. It is therefore important to elucidate the behaviour of instanton effects across such loci, and the mechanisms to restore holomorphy of the $4 \mathrm{~d}$ non-perturbative F-term.

This program was initiated in [28] for instantons generating superpotential terms, and crossing lines of threshold stability, ${ }^{1}$ namely real codimension one loci in moduli space, where a BPS brane splits into two (or more) mutually BPS branes, which beyond the line recombine back into another single BPS brane. Continuity of the superpotential requires novel kinds of contributions to the non-perturbative superpotential arising from multiinstanton effects. These multi-instanton effects have subsequently appeared in several new applications and contexts [30, 24, 25].

Among the systems considered in [28], there is no example of BPS instantons contributing to the superpotential and crossing genuine lines of marginal stability, i.e. codimension one loci in moduli space, beyond which a BPS instanton turns into a (possibly multi-instanton) non-BPS system. In fact, such a situation is not possible, for a simple well-known reason. For an instanton to contribute to the superpotential, it must have exactly two fermion zero modes (to saturate the $d^{2} \theta$ superspace integration); while a non-BPS instanton breaks all supersymmetries and therefore has at least four goldstino fermion zero modes (which saturate the $d^{4} \theta$ superspace integration, so the instanton actually generates a $4 \mathrm{~d}$ D-term). This simple observation, together with continuity of the non-perturbative effects, implies that it is not possible that a BPS instanton contributing to the superpotential is connected with a non-BPS instanton. ${ }^{2}$

Equivalently, any BPS instanton which can cross a genuine line of marginal stability and become non-BPS cannot contribute to the non-perturbative superpotential. Such BPS instantons must have additional fermion zero modes, and therefore contribute to multifermion F-terms (denoted higher F-terms henceforth). The present paper is devoted to the study of the behaviour of non-perturbative higher F-terms across lines of marginal stability for the underlying instantons. Since higher F-terms are chiral operators and enjoy interesting holomorphy properties, they are expected to be well-behaved upon such crossings.

This would seem to contradict the equally well-founded expectation that the non-BPS instanton generates a D-term. As we argue, there is no contradiction, for a simple but deep reason. As discussed in [31, 32], higher F-terms are 4d operators which, when regarded as functions over moduli space, are associated to a non-trivial class of a certain cohomology, such that locally in moduli space can be written as integrals over all superspace, but not

\footnotetext{
${ }^{1}$ We adopt the nomenclature in 29] and distinguish between lines of threshold stability and of marginal stability. We refer to both of them as lines of BPS stability, in that the spectrum of BPS objects jumps at them.

${ }^{2}$ While this paper was in preparation, a revised version of [25] appeared, with results consistent with our statement.
} 
globally in moduli space.

Thus the instanton amplitude is in such a cohomologically non-trivial class, when regarded globally in moduli space. Away from the BPS locus, the instanton amplitude is locally trivial in moduli space, and can be written as an integral over all superspace, a $4 \mathrm{~d}$ D-term, in agreement with standard wisdom for non-BPS instantons. On the BPS locus, the amplitude reduces to a chiral operator integrated over half superspace, an F-term, in agreement with standard wisdom for BPS instantons. In this sense, the cohomological obstruction to writing the amplitude as a globally defined D-term localizes on the BPS locus. Continuity and holomorphy of the $4 \mathrm{~d}$ instanton amplitude are naturally described using these concepts.

We can also phrase in this language the fact that BPS instantons generating superpotentials cannot become non-BPS. A superpotential is non-trivial even locally in moduli space, in the sense that it cannot be written as an integral over all superspace. Therefore, a putative non-BPS instanton generating a superpotential would contradict standard wisdom of instanton physics, since such superpotential cannot be written as a D-term, even locally in moduli space.

The fact that only instantons with additional fermion zero modes can cross genuine lines of marginal stability (while instantons with two fermion zero modes have at most lines of threshold stability) leads to an interesting puzzle. Indeed, there are different mechanisms like, e.g. closed string fluxes, which can lift additional fermion zero modes and turn one kind of instanton into another. The resolution involves four-dimensional supersymmetry breaking in an interesting way. Consider a BPS instanton which can become non-BPS, and which therefore has additional fermion zero modes. Any mechanism which lifts the additional fermion zero modes and makes the instanton contribute to the superpotential (at least on the BPS locus), simultaneously triggers $4 \mathrm{~d}$ supersymmetry breaking in the region of moduli space where the instanton is non-BPS. Since there is no supersymmetry in the background in this region, the instanton need not have four goldstino zero modes, thus avoiding a contradiction with the fact that the would-be goldstinos have been lifted. We present several realizations of this general argument, providing further support for our general picture.

As we have mentioned, the prototypical example of line of marginal stability is provided by a BPS instanton, which splits into several BPS instantons, which subsequently misalign their BPS phases and define an overall non-BPS system. The discussion of how a multiinstanton process reconstructs the amplitude of a single-instanton one is similar to [28], and we will not pursue it further here. Indeed, most of the new conceptual issues are related to the instanton becoming a non-BPS system, rather than to its splitting. Hence, it is more illustrative to focus on simpler systems of instantons which are BPS on a real codimension one locus in moduli space, and are non-BPS away from it, with no splitting whatsoever. We regard this situation as another kind of line of BPS stability, with many features in common to lines of marginal stability. Indeed, as we discuss in one example, the resulting lessons, complemented with ideas in [28], suffice to describe lines of marginal stability with splitting of instantons.

The paper is organized as follows. In section 2 we review some useful properties of 
higher F-terms. In section 3 we describe the example of an isolated U(1) instanton, with a real codimension one BPS locus in moduli space, away from which it is non-BPS due to a misalignment of its calibrating phase. We describe the structure of its amplitude on the BPS locus, in the deep non-BPS regime, and their nice continuity in the near-BPS region. We show in section 3.6 that these concepts carry over with little modification to lines of marginal stability involving splitting of instantons. In section 1 we discuss the instanton amplitude and a line of BPS stability in a system providing a D-brane realization of $N_{f}=N_{c}$ SQCD. In section 5 we present examples showing the correlation between fermion zero mode lifting and $4 \mathrm{~d}$ supersymmetry breaking. Finally, section 6 contains some concluding remarks and outlook.

\section{Review of higher F-terms}

In this section we briefly review some useful properties of multi-fermion F-terms (higher F-terms henceforth), following [31, 32].

BPS instantons with $2 p$ additional fermion zero modes, beyond the two $\mathcal{N}=1$ goldstinos, generate a multi-fermion F-term of the form

$$
\begin{aligned}
\delta S & =\int d^{4} x d^{2} \theta \omega_{\bar{i}_{1} \ldots \bar{i}_{p} \bar{j}_{1} \cdots \bar{j}_{p}}(\Phi)\left(\bar{D}_{\dot{\alpha}_{1}} \bar{\Phi}^{\bar{i}_{1}} \bar{D}^{\dot{\alpha}_{1}} \bar{\Phi}^{\bar{j}_{1}}\right) \cdots\left(\bar{D}_{\dot{\alpha}_{p}} \bar{\Phi}^{\bar{i}_{p}} \bar{D}^{\dot{\alpha}_{p}} \bar{\Phi}^{\bar{j}_{p}}\right), \\
& \equiv \int d^{4} x d^{2} \theta \mathcal{O}_{\omega}
\end{aligned}
$$

where the field dependent tensor $\omega_{\bar{i}_{1} \cdots \bar{i}_{p}} \bar{j}_{1} \cdots \bar{j}_{p}$ is antisymmetric in the $\bar{i}_{k}$ and also in the $\bar{j}_{k}$, and symmetric under their exchange. Formally it can be regarded as a section of $\bar{\Omega}_{\mathcal{M}}^{p} \otimes \bar{\Omega}_{\mathcal{M}}^{p}$.

The conditions that $\delta S$ is supersymmetric and a non-trivial F-term implies that $\omega$ belongs to a non-trivial cohomology class in moduli space, for the non-standard cohomology defined below. The condition that $\delta S$ is supersymmetric is that $\mathcal{O}_{\omega}$ is chiral, namely annihilated by the supercharges $\bar{Q}_{\dot{\alpha}}$. This requires that $\omega$ is holomorphic, namely closed under $\bar{\partial}$. On other hand, even if $\delta S$ is supersymmetric, it may represent a trivial $F$-term. Though written in (2.1) in the form $\int d^{2} \theta(\ldots)$, it may be that $\delta S$ can be alternatively written $\int d^{4} \theta(\ldots)$, in other words as a $D$-term. In fact, if $\mathcal{O}_{\omega}=\left\{\bar{Q}_{\dot{\alpha}},\left[\bar{Q}^{\dot{\alpha}}, V\right]\right\}$ for some $V$, then $\mathcal{O}$ is trivially chiral and one can write $\delta S=\int d^{4} x d^{4} \theta V$. One must therefore impose an equivalence relation on the space of operators $\mathcal{O}_{\omega}$, under which it is considered trivial if $\delta S$ is equivalent to a $D$-term. The equivalence relation is of the form

$$
\omega_{\bar{i}_{1} \cdots \bar{i}_{p} \bar{j}_{1} \cdots \bar{j}_{p}} \sim \omega_{\bar{i}_{1} \cdots \bar{i}_{p} \bar{j}_{1} \cdots \bar{j}_{p}}+\nabla_{\left[\bar{i}_{1}\right.} \xi_{\left.\bar{i}_{2} \cdots \bar{i}_{p}\right] \bar{j}_{1} \cdots \bar{j}_{p}}+\left(\bar{i}_{k} \leftrightarrow \bar{j}_{k}\right) .
$$

with symmetrization of the $i$ and $j$ indices in the term involving $\xi$. This condition is actually somewhat stronger in principle than being a D-term, and just requires that $\delta S$ cannot be written as an integral over $3 / 4$ of superspace.

As a simple example, consider the familiar statement that any correction $\delta K$ to the Kähler form can be trivially rewritten as an $F$-term correction upon performing half the integral over superspace:

$$
\int d^{4} x d^{4} \theta \delta K=\int d^{4} x d^{2} \theta \bar{D}^{2} \delta K=\int d^{4} x d^{2} \theta \nabla_{\bar{i}} \nabla_{\bar{j}} \delta K\left(\bar{D} \bar{\Phi}^{\bar{i}} \cdot \bar{D} \bar{\Phi}^{\bar{j}}\right)
$$


where $\nabla$ is the covariant derivative in field space. Hence 4-fermion F-terms with $\omega_{\bar{i} \bar{j}}=$ $\nabla_{\bar{i}} \nabla_{\bar{j}} \delta K$ are trivial and actually correspond to D-terms.

The condition that $\omega$ defines a supersymmetric operator (i.e. closed under $\bar{\partial}$ ) but a nontrivial F-term (i.e. not exact in the above sense) implies that it defines a non-trivial class with respect to a cohomology. Because of the symmetrization in (2.2), the cohomology does not correspond to the usual Dolbeault cohomology. Still, the only information we need to keep in mind for our purposes is that the cohomology groups associated to higher F-terms are locally trivial in moduli space. Namely, it is always possible to write the interaction as a D-term locally in moduli space, but there may be a cohomological obstruction to doing it globally in moduli space. The interaction must therefore be written as an F-term of the form (2.1), modulo pure D-terms.

\section{The isolated $U(1)$ instanton}

In this section we consider the simplest system of an instanton which can become nonBPS, by simple misalignment of its BPS phase. As argued in the introduction, it cannot contribute to the superpotential but rather to higher F-terms of the kind studied in previous section. This example of line of BPS stability is simple in that the instanton does not split, but still leads to several important lessons, which apply with little modification to more involved lines of marginal stability where instantons split, as we show in section 3.6.

\subsection{The setup}

Let us consider a Calabi-Yau compactification with an orientifold projection, and a D-brane instanton which is not mapped to itself under it (we denote the instanton $\mathrm{U}(1)$ hereafter). We consider the instanton to be described by an A- or B- brane in type IIA or type IIB respectively, so that the holomorphic part of the supersymmetry conditions are satisfied. Such instanton has a real codimension one BPS locus in moduli space (complex structure in IIA, Kahler in IIB), where its BPS phase aligns with the $\mathcal{N}=1$ subalgebra preserved by the orientifold plane. At this locus the instanton breaks half of the supersymmetries of the $4 \mathrm{~d} \mathcal{N}=1$ background, while away from it it breaks all supersymmetries. Finally, for simplicity we consider the instanton to be rigid, and isolated, i.e. not intersecting its orientifold image, so that the zero mode structure reduces to the universal sector of four translational bosonic zero modes and four fermionic zero modes.

It is easy to devise simple realizations using euclidean D2-branes wrapped on 3-cycles in a IIA orientifold compactification, and we phrase the discussion in these terms (although it is straightforward to do it for general situations in IIB). For a fully explicit realization, we can use the geometries described in appendix A, see figure 1.

Let us fix some notation for the relevant closed string moduli. Consider a D2-brane wrapped on a 3 -cycle $A$ and its orientifold image wrapped on $A^{\prime}$. We also introduce the dual cycles $B$ and $B^{\prime}$. Before the orientifold projection, the relevant closed string sector is given by two hypermultiplets, with $4 \mathrm{~d}$ bosonic components

$$
t=\int_{A} \operatorname{Re} \Omega+i \int_{A} C_{3}, \quad u=\int_{B} \operatorname{Re} \Omega+i \int_{B} C_{3}
$$


a)

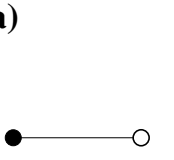

b)

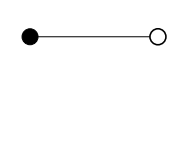

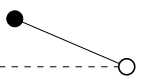



Figure 1: A rigid isolated U(1) instanton (a) on the BPS locus (with respect to the $\mathcal{N}=1$ supersymmetry preferred by the orientifold plane, shown as a dashed line), and (b) when non-BPS due to a misalignment of its BPS phase. As usual, black and white dots denote the degenerations of the double $\mathbf{C}^{*}$ fibration in the geometries discussed in appendix $\mathrm{A}$.

\begin{tabular}{|c|c|}
\hline $\mathcal{N}=1$ & $\mathcal{N}=1^{\prime}$ \\
\hline$\theta^{\alpha}$ & $\tau^{\alpha}$ \\
\hline $\bar{\theta}^{\alpha}$ & $\bar{\tau}^{\dot{\alpha}}$ \\
\hline
\end{tabular}

Table 1: Universal fermionic zero modes $\theta^{\alpha}, \bar{\tau}^{\dot{\alpha}}$ of an instanton associated with the breaking of an $\{\mathcal{N}=2\}=\{\mathcal{N}=1\} \oplus\left\{\mathcal{N}=1^{\prime}\right\}$ SUSY algebra into the $\mathcal{N}=1$ subalgebra preserved by the orientifold. For an anti-instanton the universal zero modes correspond to $\left(\tau^{\alpha}, \bar{\theta}^{\dot{\alpha}}\right)$.

and similar primed fields for the primed cycles. In $4 \mathrm{~d} \mathcal{N}=1$ terms we have the chiral multiplets

$$
T=t+\theta \psi \quad, \quad \Sigma=u+\theta \chi
$$

and similar primed fields. The combinations invariant under the orientifold are $T+T^{\prime}$ and $\Sigma-\Sigma^{\prime}$, which we also denote $T, \Sigma$ by abuse of language. For future convenience, we also define

$$
\xi=\operatorname{Re} u=\int_{B} \operatorname{Re} \Omega
$$

\subsection{On the BPS locus}

Let us consider the BPS locus, where the instanton is BPS with respect to the $\mathcal{N}=1$ preferred by the orientifold plane, see figure 1 1a. As mentioned, there are only the four zero modes, which we denote $\theta, \bar{\tau}$, providing the goldstinos of the $\mathcal{N}=2$ susy in the absence of the orientifold plane. The modes $\theta$ are goldstinos of the $\mathcal{N}=1$ supersymmetry of the compactification, and are saturated by the $d^{2} \theta$ integration over half of superspace of the non-perturbative 4d F-term expected from a BPS instanton. The modes $\bar{\tau}$ are additional fermion zero modes, would-be goldstinos of an orthogonal $\mathcal{N}=1^{\prime}$ broken by the orientifold plane. Their presence implies that the F-term is not a superpotential, but rather a higher F-term.

Higher F-terms from D-brane instantons have been considered e.g. in [33]. Let us nevertheless offer a heuristic derivation of the structure of the amplitude, well suited for our purposes. Since it is necessary to integrate over the instanton fermion zero modes, one needs to write the instanton action with its full dependence on $\theta, \bar{\tau}$. Namely, one 
needs to include the supersymmetric variations of the instanton action with respect to the corresponding supersymmetries. We thus have

$$
S_{\mathrm{inst}}=t+\theta \delta_{\theta} t+\bar{\tau} \bar{\delta}_{\bar{\tau}} t+\left(\theta \delta_{\theta}\right)\left(\bar{\tau} \bar{\delta}_{\bar{\tau}}\right) t
$$

where the $\delta$ 's denote the variations with respect to the $\mathcal{N}=2$ supersymmetries. Namely

$$
\delta_{\theta} t=\psi \quad, \quad \bar{\delta}_{\bar{\tau}} t=\bar{\chi} \quad, \quad \delta_{\theta^{\alpha}} \bar{\delta}_{\bar{\tau}^{\dot{\alpha}}} t=\left(\bar{\sigma}^{\mu}\right)_{\alpha \dot{\alpha}} \partial_{\mu} u
$$

where the last expression follows from the observation that $\bar{\chi}$ is the lowest component of the chiral multiplet

$$
\bar{D}_{\dot{\alpha}} \bar{\Sigma}=\bar{\chi}_{\dot{\alpha}}+\left(\theta \bar{\sigma}^{\mu}\right)_{\dot{\alpha}} \partial_{\mu} u
$$

Notice that the value of the parameter $\xi$ does not appear in this action (only its derivatives do). ${ }^{3}$ Indeed, it controls the misalignment with respect to the $\mathcal{N}=1$ supersymmetry, so it is implicitly set to zero in the above expression in order to keep our instanton BPS. Note also that we are already implicitly using supermultiplets under the $\mathcal{N}=1$ supersymmetry preferred by the orientifold, and that the above discussion can be carried out similarly for the orientifold image. Including the orientifold and its image, the complete instanton amplitude reads

$$
\int d^{2} \theta d^{2} \bar{\tau} e^{-(T+\bar{\tau} \overline{D \Sigma})}=\int d^{2} \theta e^{-T} \overline{D \Sigma} \cdot \overline{D \Sigma}
$$

This is a supersymmetric higher F-term, due to holomorphy of the coefficient. Its nontriviality is encoded in the fact that the instanton is BPS and has no fermion zero modes along the $\bar{\theta}$ direction in superspace.

\subsection{Away from the BPS locus}

Let us move the system away from the BPS locus by turning on the real closed string modulus Re $u$ in the multiplet $\Sigma$, which in the IIA realization controls the size of the 3-cycle B. As is manifest in figure 1 $\mathrm{b}$, where it corresponds to moving the white degeneration away from the real axis, this modulus controls the misalignment of the BPS phase of the instanton.

In general, such misaligned instantons have a very non-holomorphic structure. For instance, the bosonic part of their classical action (the wrapped volume of the instanton and its orientifold image) is a non-holomorphic function of the moduli of the $\mathcal{N}=1$ theory. To be more specific, consider the IIA setup, with a D2-brane instanton wrapped on a 3cycle calibrated with respect to $e^{i \xi} \Omega$, where $\xi$ is the phase of the central charge. ${ }^{4}$ The real part of the classical action of such instanton is given by $\left.\operatorname{Re}\left(e^{i \xi} \Omega\right)\right|_{\Pi}$. This can be phrased

\footnotetext{
${ }^{3}$ Incidentally, one can also heuristically understand the derivative coupling $u$, or rather its imaginary part, as follows. The field $\operatorname{Im} u$ is related to the integral of RR potentials over the wrapped cycle, so $\theta \bar{\sigma}^{\mu} \bar{\tau} \partial_{\mu} u$ comes from the coupling of fermionic fields to RR field strength in the D-brane fermionic action (see e.g. 34]).

${ }^{4}$ Abusing language, we use the same notation for the BPS phase and for Re $u$, even though the are the same only in the near BPS regime of next section.
} 
in terms of its classical action on the BPS locus, $\left.\operatorname{Re} \Omega\right|_{\Pi}$, which is a holomorphic function of the moduli, by using the calibration condition

$$
\left.\operatorname{Im}\left(e^{i \xi} \Omega\right)\right|_{\Pi}=\left.0 \quad \longrightarrow \quad \operatorname{Im} \Omega\right|_{\Pi}=-\left.\tan \xi \operatorname{Re} \Omega\right|_{\Pi}
$$

so that

$$
\left.\operatorname{Re}\left(e^{i \xi} \Omega\right)\right|_{\Pi}=\left.\cos \xi \operatorname{Re} \Omega\right|_{\Pi}-\left.\sin \xi \operatorname{Im} \Omega\right|_{\Pi}=\frac{\left.\operatorname{Re} \Omega\right|_{\Pi}}{\cos \xi}
$$

which is a non-holomorphic function of the moduli, since $\xi$ is real.

A second general feature of these non-BPS instantons is that they have at least four exact fermion zero modes, the goldstinos generated by acting on the instanton with the four supercharges it breaks. Hence, in agreement with standard wisdom, such non-BPS instantons generate D-terms. Indeed, we can be more explicit in this respect, by considering the structure of fermion zero modes for a non-BPS instanton with a non-zero, constant phase $\xi$. The instanton is supersymmetric with respect to the $\mathcal{N}=1$ subalgebra of the underlying $\mathcal{N}=2$ defined by the phase $\xi$. The fermion zero modes, denoted $\theta^{\prime}$ and $\bar{\tau}^{\prime}$, are associated to the orthogonal supercharges. This primed modes can be rewritten in terms of the unprimed modes of the reference $\mathcal{N}=1$ subalgebra by performing a spinorial rotation of angle $\xi$ :

$$
\begin{aligned}
\bar{\tau}^{\prime} & =\cos (\xi / 2) \bar{\tau}+\sin (\xi / 2) \bar{\theta} \\
\theta^{\prime} & =\cos (\xi / 2) \theta+\sin (\xi / 2) \tau
\end{aligned}
$$

This expression can be understood easily by noticing that the preserved goldstinos for a rotated brane are solutions of the equation [35]:

$$
\varepsilon_{L}=R \Gamma_{\mathrm{Dp}} R^{-1} \varepsilon_{R}
$$

where $\Gamma_{\mathrm{Dp}}$ is a product of $\Gamma$-matrices along the worldvolume of the unrotated brane and $R$ is the rotation matrix relating the rotated system to the unrotated one. This equation implies that the susys preserved by the rotated instanton are simply a rotation of the solutions for the aligned instanton, as in (3.10).

In the computation of the instanton amplitude, the fermion zero mode integration measure $d^{2} \theta^{\prime} d^{2} \bar{\tau}^{\prime}$ can be expressed in terms of variables natural in the $4 \mathrm{~d} \mathcal{N}=1$ supersymmetry of the compactification, by using ${ }^{5} d^{2} \bar{\tau}^{\prime}=\sin ^{2}(\xi / 2) d^{2} \bar{\theta}$ (and similarly $d^{2} \theta^{\prime}=\cos ^{2}(\xi / 2) d^{2} \theta$ ). Thus for non-zero BPS phase, the instanton generate terms which can be written as integrals over all of superspace $\int d^{2} \theta d^{2} \bar{\theta}(\ldots)$, so the non-BPS instanton generates a D-term.

However, regarding the instanton induced $4 \mathrm{~d}$ operator as a global function over moduli space, the above change of variables signals a pathology at the BPS locus, where $\xi=0$. The goldstino $\bar{\tau}$ has zero component along $\bar{\theta}$, and therefore the instanton effect cannot be written as a D-term on this locus. In the language of section 2, the D-term produced by

\footnotetext{
${ }^{5} \mathrm{An}$ analogue for bosonic variables is to consider a 2-plane parameterized by $x, y$, and introduce a system rotated by an angle $\xi / 2$, namely $x^{\prime}=\cos (\xi / 2) x+\sin (\xi / 2) y, y^{\prime}=-\sin (\xi / 2) x+\cos (\xi / 2) y$. An integral along the $x^{\prime}$ axis (hence $y^{\prime}=0$ and so $y=\tan (\xi / 2) x$ ) can be traded to an integral over $y$ using $d x^{\prime}=d y / \sin (\xi / 2)$.
} 
the instanton is in a non-trivial class of the Beasley-Witten cohomology, with the cohomological obstruction localized on the BPS locus, where the contribution must be written as a genuine F-term.

This observation, to be developed in the next paragraph, underlies the continuity of the non-perturbative contribution across the BPS line. The generation of the D-term away from the BPS locus, as dictated by physics of non-supersymmetric instantons, does not contradict the generation of an F-term at the BPS locus, as dictated by physics of supersymmetric instantons.

\subsection{The near BPS regime}

In order to show that the D-term generated by the non-BPS instanton reduces to the required F-term at the BPS locus, we consider the instanton slightly away from the BPS locus. Treating the amplitude in an expansion in $\xi$, the $\mathcal{O}\left(\xi^{0}\right)$ piece reduces to the genuine F-term of the BPS locus. Higher order contributions should correspond to globally defined D-terms.

Consider moving slightly away from the BPS locus by moving in closed string moduli space in the direction $\xi$. From the viewpoint of the instanton, this corresponds, in first approximation, to turning on a world-volume Fayet-Iliopoulos term. Thus at leading order the interesting couplings on the instanton world-volume theory are

$$
\xi D ; \overline{\chi \tau}
$$

These can be understood by analogy with the terms obtained from coupling a fielddependent FI term in $4 \mathrm{~d} \int d^{4} \theta(\Sigma+\bar{\Sigma}) V$. The first term, once the auxiliary field $D$ is integrated out gives rise to an $\mathcal{O}\left(\xi^{2}\right)$ term, corresponding to the quadratic term in the expansion of the non-BPS instanton classical action $\left.\operatorname{Re} \Omega\right|_{\Pi} / \cos \xi$. We drop it in our $\mathcal{O}\left(\xi^{0}\right)$ computation. Notice also that the fermion zero modes $\theta^{\prime}, \bar{\tau}$, reduce to $\theta, \bar{\tau}$ at this order.

The full instanton action, completing the $\theta$-dependence on the second term in (3.12) (i.e. promoting $\bar{\chi}$ to the $4 \mathrm{~d}$ supermultiplet $\overline{D \Sigma}$ ) is

$$
S_{\text {inst }}=T+\bar{\tau} \overline{D \Sigma}
$$

thus reproducing the contribution on the BPS locus, which arises as a manifest F-term.

$$
\int d^{4} x d^{2} \theta d^{2} \bar{\tau} e^{-(T+\bar{\tau} \overline{D \Sigma})}=\int d^{4} x d^{2} \theta e^{-T} \overline{D \Sigma} \overline{D \Sigma}
$$

If preferred, in the above analysis we could have kept the integration over $\bar{\theta}$ instead of $\bar{\tau}$, in order to make the connection with the situation far away from the BPS locus. The result is the same, simply noticing the cancellations between $\xi$-dependent factors (to the corresponding order) between the fermion zero mode measure, and the insertions required to saturate $\bar{\theta}$.

In conclusion, the complete instanton amplitude reduces to the above F-term on the BPS locus. Thus, the complete instanton amplitude and the F-term on the BPS locus correspond to F-terms in the same Beasley-Witten cohomology class. This implies that the higher order terms in $\xi$ correspond to pure D-terms, which are globally defined over moduli space, and that the genuine F-term contribution of the instanton is given by the above expression. 


\subsection{The global picture}

Let us recap and refine the global picture. The instanton generates an F-term contribution in a cohomologically non-trivial class of the Beasley-Witten cohomology. This F-term is trivial away from the BPS locus and can be expressed as an integral over all superspace in agreement with the standard wisdom that non-BPS instantons generate non-perturbative D-terms. The instanton amplitude on the BPS locus is given by (3.7), and defines a genuine F-term, in agreement with the standard wisdom for BPS instantons.

Let us comment on a possible source of confusion, regarding the holomorphy of the instanton induced F-term as a function of moduli, in particular of moduli which take the instantons away from their BPS locus. Clearly the complete instanton amplitude does not depend holomorphically on the $4 \mathrm{~d}$ moduli, in particular on the moduli which take the instantons away from their BPS locus. On the other hand, the BPS amplitude (3.7) does have a nice holomorphic structure, but, extending it trivially to the complete moduli space, is not the complete expression for the instanton amplitude away from the BPS locus. The crucial point is that both expressions are two representatives of the same cohomology class, and thus differ by a globally defined D-term. Therefore, all the non-holomorphies in the complete instanton amplitude can be assigned to the part that corresponds to a globally defined D-term. The genuine F-term is therefore holomorphic in all moduli, and in particular (3.7) provides a particular representative which makes this property manifest. It is in this sense that holomorphy of the non-perturbative F-term from the instanton is preserved across the line of BPS stability. Although we phrased the discussion in terms of our particularly simple example, the argument applies in full generality to any other situations, including instantons that split at lines of marginal stability, as we argue in the next section.

\subsection{Application to lines of marginal stability}

In order to show that the lesson learned in the previous section applies also to instantons that split at lines of marginal stability, we discuss the simplest situation of this kind. Using a type IIA language, consider a system of two rigid U(1) instantons, wrapped on two cycles $A_{1}, A_{2}$, with one chiral intersection, and away from the orientifold planes so that they do not intersect the orientifold image of the system.

Such systems are easy to engineer in toroidal orientifold examples. Also, although the geometries in appendix A realize naturally only non-chiral intersections, it is straightforward to construct examples with such chiral intersections in closely related geometries. Consider a complex plane $z$ over which we fiber a $\mathbf{C}^{*}$ degenerating at points labeled $z=a_{i}$, times an elliptic fiber, with $\left(p_{i}, q_{i}\right) 1$-cycles degenerating at points labeled $z=b_{i}$. Our system of interest can be realized by considering a geometry with one $a$-type degeneration and two $b$-type degenerations, with $\left(p_{1}, q_{1}\right)=(1,0),\left(p_{2}, q_{2}\right)=(0,1)$. The two relevant 3 -cycles $C_{1}, C_{2}$ are obtained by considering segments on the $z$-plane joining $\left[a, b_{1}\right]$, and $\left[a, b_{2}\right]$, respectively, and over which we fiber the circle in $\mathbf{C}^{*}$ fiber and the 1-cycles $(1,0)$ and $(0,1)$ on the elliptic fiber, respectively. The 3 -cycles have intersection numbers $\left[C_{1}\right] \cdot\left[C_{2}\right]=1$. By locating the degenerations in suitable locations on the $z$-plane the systems can be taken 
to be BPS, or misaligned by an arbitrary amount. It is also straightforward to add orientifold planes and other required ingredients. Incidentally, the above double fibrations have appeared as the mirrors of systems of D-branes at singularities, see e.g. [36, 37]. In this language, the above realization of our system can be regarded as considering two fractional branes on the complex cone over $d P_{1}$. In any event, we can proceed with our discussion in full generality, independently of the details of specific realizations.

The zero mode content of the instanton system is given by the two universal sets $x_{i}^{\mu}$, $\theta_{i}, \bar{\tau}_{i}$, for $i=1,2$, and bosonic and fermionic zero modes at the instanton intersection $m$, $\psi$, and $\bar{m}, \bar{\psi}$, with charges $(+1,-1)$ and $(-1,+1)$ under the two $\mathrm{U}(1)$ 's.

The system has a line of marginal stability, which is controlled by a real parameter. For simplicity we may keep the 3 -cycle $A_{2}$ aligned, then the relevant real parameter is $\xi=\int_{B_{1}} \operatorname{Im} \Omega$, with an additional term $-\int_{B_{1}^{\prime}} \operatorname{Im} \Omega$ if we include the orientifold image system. ${ }^{6}$ The line of marginal stability is located at $\xi=0$. We focus on its neighbourhood, namely small $\xi$, where its effect can be described as a world-volume FI term for U(1) ${ }_{1}$. The discussion of the world-volume action in this regime can be carried out similar to [28]. We have the couplings

$$
S_{2-\text { inst }}=\left|x_{1}-x_{2}\right|^{2}|m|^{2}+i\left(x_{1}^{\mu}-x_{2}^{\mu}\right) \bar{\psi} \sigma_{\mu} \psi+\psi\left(\theta_{1}-\theta_{2}\right) \bar{m}+\bar{\psi}\left(\bar{\tau}_{1}-\bar{\tau}_{2}\right) m
$$

In addition, there are couplings involving fields in the FI multiplet $\Sigma$

$$
V_{D}=(m \bar{m}-\xi)^{2} \quad ; \quad S_{\bar{\tau}_{1}}=\bar{\tau}_{1} \overline{D \Sigma}
$$

Consider the system for $\xi>0$ which corresponds to the BPS side. Heuristically, the bosons $m, \bar{m}$ acquire a vev to minimize the scalar potential $V_{D}$. The instantons recombine into a single rigid BPS U(1) instanton. The vev accordingly also freezes the two instantons at the same position $x_{1}=x_{2}$ in space, and in the fermionic coordinates $\theta_{1}=\theta_{2}, \tau_{1}=\tau_{2}$, by making massive their difference fields (along with $\psi, \bar{\psi}$ ). We are left with a recombined BPS instanton with a universal sector of translational zero modes $x_{1}-x_{2}$, and fermion zero modes $\theta=\theta_{1}+\theta_{2}, \bar{\tau}=\bar{\tau}_{1}+\bar{\tau}_{2}$. The latter pull out two insertions of $\overline{D \Sigma}$, so that, denoting $T=T_{1}+T_{2}$, the instanton amplitude is

$$
\int d^{4} x d^{2} \theta e^{-T} \overline{D \Sigma} \overline{D \Sigma}
$$

as expected for the recombined BPS rigid instanton (notice that $\Sigma$ can be regarded as controlling the 3 -cycle dual to $A=A_{1}+A_{2}$ ). In a more proper treatment, $m, \bar{m}$ are not frozen at their vevs, but rather one integrates over these bosonic modes. The above heuristic computation, however, provides the saddle point approximation to the instanton computation.

At the BPS locus $\xi=0$, we have a system of two mutually BPS instantons, and the amplitude (3.17) is reconstructed by a 2-instanton process involving both. In this computation, the above saddle point approximation is not valid (since it would fix $m=0$ and all fermion interactions would disappear), but the correct computation by integrating

\footnotetext{
${ }^{6}$ In more involved situations with two parameters $\xi_{1}, \xi_{2}$, the same role is played by $\xi=\xi_{1}-\xi_{2}$.
} 
over bosonic zero modes allows to keep the interactions and saturate all fermion zero modes just as in the above discussion. Hence, one recovers the same F-term structure (3.17).

Finally, for $\xi<0$ it is not possible to cancel the world-volume D-term scalar potential, and we have a system of two instantons with different BPS phases, thus defining an overall non-BPS system. The non-cancellation of $V_{D}$ reflects the non-holomorphic dependence of the total wrapped volume on the moduli. Nevertheless, the discussion of fermion zero mode saturation is exactly as in the previous single instanton situation. We are left with a non-BPS instanton, with fermion zero modes $\theta=\theta_{1}+\theta_{2}+\mathcal{O}(\xi)$ and $\bar{\tau}=\bar{\tau}_{1}+\bar{\tau}_{2}+\sin (\xi / 2) \bar{\theta}$. The fact that $\bar{\tau}$ picks up a component along $\bar{\theta}$ allows to write the instanton amplitude as a D-term, as expected for a non-BPS instanton, with no discontinuity at the BPS locus, since the non-BPS instanton amplitude reproduces the F-term (3.17) from its $\mathcal{O}\left(\xi^{0}\right)$ contribution.

As announced, the main concepts involved in the study of instantons at lines of marginal stability can be already obtained from simpler systems with lines of BPS stability where instantons do not split. We therefore focus on the latter kind of systems to continue discussing other new features, with the understanding that their discussion in genuine lines of marginal stability is possible, and very similar.

\section{The $N_{f}=N_{c}$ SQCD instanton from branes}

In the above discussion, the zero modes $\bar{\tau}$ played a fundamental role, in that they allowed an interpolation between fermion zero modes with the interpretation of goldstinos $\bar{\theta}$ (away from the BPS locus) and extra fermion zero modes making the non-perturbative contribution a higher F-term rather than a superpotential (at the BPS locus). It would seem crucial that the $\bar{\tau}$ are unlifted, and that the argument does not apply to instantons where these models are lifted by interactions. In this section we argue that in fact the discussion continues to be valid in such cases. We describe this in an illustrative example, the D-brane realization of the $N_{c}=N_{f}$ SQCD instanton. Since this example provides a stringy ${ }^{7}$ realization of the well-studied system in [31] (modulo the gauging of the center of mass U(1) in our case), our discussion is sketchy and targeted to illustrating the physics of the modes $\bar{\tau}$. In fact we carry out most computations in the case $N_{f}=N_{c}=1$, where there is no classical field theory interpretation for the instanton (it is stringy in the sense of [38]), since the latter is not essential to our purpose.

\subsection{The setup}

Consider a D-brane instanton with the interpretation of a gauge instanton in an $N_{f}=N_{c}$ SQCD theory. Namely, in the IIA setup we consider $N_{c}=N$ D6-branes wrapped on an rigid 3-cycle $A$, and $N_{f}=N$ D6-branes wrapped on a (possibly non-compact) 3-cycle $A_{\text {flav. }}$, with a non-chiral intersection with $A$. The instanton of interest is a D2-brane wrapped on $A$. The setup is easy to engineer using the geometries described in appendix A, by wrapping

\footnotetext{
${ }^{7}$ For a recent detailed study of the instanton in $N_{f}=N_{c}$ SQCD in a string theory realization, see [26].
} 
a)



b)

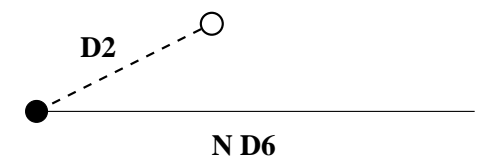

Figure 2: D-brane realization of the $N_{f}=N_{c}$ SQCD theory in a geometry of the kind in appendix A. The construction shows that instanton on its BPS locus (a) and away from it (b). Notice that gauge D-branes recombine and remain BPS through the process.

\begin{tabular}{|c|c|c|c|}
\hline Field & $\mathrm{U}(1)_{\text {inst }}$ & $\mathrm{U}\left(N_{c}\right)$ & $\mathrm{U}\left(N_{f}\right)$ \\
\hline$m, \beta$ & +1 & $\bar{\square}$ & \\
\hline $\bar{m}, \bar{\beta}$ & -1 & $\square$ & \\
\hline$\tilde{\eta}$ & -1 & & $\square$ \\
\hline$\tilde{\eta}$ & +1 & & $\bar{\square}$ \\
\hline$Q$ & & $\square$ & $\bar{\square}$ \\
\hline$\tilde{Q}$ & & $\bar{\square}$ & $\square$ \\
\hline
\end{tabular}

Table 2: Zero modes between the different branes in the system with their representations.

sets of $N$ D6-branes on a compact and a non-compact 3-cycles as shown in figure $2{ }^{8}$ As in previous examples, there is a real parameter, given by the size of the 3 -cycle $B$ dual to $A$, which controls the misalignment of the instanton, see figure $2 \mathrm{~b}$. Note that the misalignment of the instanton does not imply a misalignment of the gauge D-branes, since the latter can recombine and remain BPS throughout moduli space. This recombination is important, as we will recall in section 4.3 .

\subsection{At the BPS locus}

The Beasley-Witten higher F-term for $N_{c}=N_{f}=1$. Let us sketch the fermion zero mode structure and their saturation in the BPS case. In addition to the four universal fermion zero modes $\theta, \bar{\tau}$, there are bosonic and fermionic zero modes charged under the instanton world-volume $\mathrm{U}(1)$. We have fermion zero modes $\beta, \bar{\beta}$ from the instanton to the color $4 \mathrm{~d}$ spacefilling D-branes, and $\eta, \bar{\eta}$ from the instanton to the flavor D-branes. In addition we have bosonic zero modes from the instanton to the color D-branes. Our description of the bosonic zero modes will be sketchy, and we simply introduce the relevant ones for fermion mode saturation which we denote by $m, \bar{m}$. The charges of these fields under the different groups are given in table 2,

where we have also included the $4 \mathrm{~d}$ quark chiral multiplets $Q=q+\theta \psi, \tilde{Q}=\tilde{q}+\theta \tilde{\psi}$. Using results in the literature e.g. [14], we have the following fermionic couplings, which

\footnotetext{
${ }^{8}$ In this case the gauge D-branes suffice to define a preferred $\mathcal{N}=1$ supersymmetry, so the presence of the orientifold plane is not necessary. If desired for any other reason, an orientifold plane could be added away from the D-brane system, along with the corresponding orientifold images.
} 
we already write in terms of $4 \mathrm{~d}$ multiplets

$$
\bar{\tau} \bar{\beta} m+\bar{\tau} \beta \bar{m}-\beta \overline{\tilde{Q}} \tilde{\eta}+\bar{\beta} \bar{Q} \bar{\eta}+\bar{m} \overline{\tilde{\eta}} \overline{D Q}+m \tilde{\eta} \overline{D \tilde{Q}}
$$

Let us focus already on the case $N_{f}=N_{c}=1$. We can saturate all the fermionic zero modes only by pulling down the first two and the last two interactions in (4.1). We obtain the analog of the Beasley-Witten result for our $N_{f}=N_{c}=1$ case

$$
|m|^{4} \overline{D Q} \overline{D \tilde{Q}}
$$

Carrying out the bosonic zero mode integral, which introduces a holomorphic function of the moduli and which we skip for simplicity, the BPS instanton amplitude has the F-term structure

$$
\int d^{2} \theta e^{-T} \overline{D Q} \overline{D \tilde{Q}}
$$

Coupling to the closed string sector. Another important coupling for our considerations involving all of moduli space is the coupling of the $\bar{\tau}$ zero modes coming from the supersymmetrization of the instanton volume:

$$
S_{\mathrm{vol}}=t+\theta \delta_{\theta} t+\bar{\tau} \overline{D \Sigma}
$$

We can use the last coupling in order to saturate some or all of the $\bar{\tau}$ zero modes. This gives rise to additional terms in the low energy effective action compared to the Beasley-Witten expression (4.3), which comes just from the open string sector couplings. For example:

$$
(\bar{\tau} \bar{\beta} m)(\bar{\tau} \overline{D \Sigma})(\beta \overline{\tilde{Q}} \tilde{\eta})(\bar{m} \overline{\tilde{\eta}} \overline{D Q}) \quad \longrightarrow \quad|m|^{2} \overline{\tilde{Q}}(\overline{D \Sigma} \overline{D Q})
$$

Saturating the fermion zero modes in all possible ways, similar to the one above, and skipping the discussion of bosonic modes, we obtain a $4 \mathrm{~d}$ instanton amplitude with the structure

$$
S_{4 d}=\int d^{4} x d^{2} \theta e^{-T}(\overline{D Q} \overline{D \tilde{Q}}+\bar{Q} \overline{D \tilde{Q}} \overline{D \Sigma}-\overline{\tilde{Q}} \overline{D Q} \bar{D} \Sigma-\bar{Q} \overline{\tilde{Q}} \overline{D \Sigma} \overline{D \Sigma})
$$

The meaning of the minus signs, and the F-term structure of this contribution will be clarified in next section. This induced operator has an interesting form, in particular it couples the open and closed string sectors in a nontrivial way. This structure can be clarified by deforming the configuration slightly away from the BPS regime, as we consider now.

\subsection{The near-BPS regime}

To address the behaviour of the system around the line of BPS stability, and also to understand better the meaning of expression (4.6), let us consider the configuration slightly away from the BPS locus of the instanton. In this regime, the misalignment can be described by the introduction of a FI term $\xi=\Sigma+\Sigma$, both in the instanton and in the 4 d gauge theory. In the latter, we can describe it as

$$
S_{\mathrm{FI}}=\int d^{4} x d^{4} \theta(\Sigma+\bar{\Sigma}) V
$$


where we ignore a possible constant coefficient. As already mentioned, in the $4 \mathrm{~d}$ gauge theory the resulting D-term can be canceled by either $Q$ or $\tilde{Q}$ acquiring a vev in order to make the abelian D-term potential vanish

$$
V_{D}=\left(|Q|^{2}-|\tilde{Q}|^{2}+\Sigma+\bar{\Sigma}\right)^{2}=0
$$

Coming back to the stringy picture, the vev for (say) $Q$ tells us to recombine the two slightly misaligned branes, and we end up in the configuration depicted in figure $2 \mathrm{~b}$.

The above discussion shows that from the string point of view, the moduli space mixes the open and closed string sectors. One should therefore find adapted coordinates which parameterize it appropriately. As is familiar, the moduli space of D-flat directions, modulo gauge transformations, can be parameterized using a set of gauge invariant operators. An important point in their construction is that the field-dependent FI term (4.7) actually arises from a modified Kahler potential for $\Sigma$ involving the vector multiplet $V$, of the form (assuming canonical Kahler potential for simplicity) 39]

$$
\int d^{4} x d^{4} \theta(\Sigma+\bar{\Sigma}-V)^{2}
$$

Hence there is a non-trivial gauge transformation of $\Sigma$ by a shift of the gauge parameter. Namely, under $V \rightarrow V+\Lambda+\bar{\Lambda}$

$$
Q \rightarrow e^{i \Lambda} Q \quad, \quad \tilde{Q} \rightarrow e^{-i \Lambda} \tilde{Q} \quad, \quad \Sigma \rightarrow \Sigma-\Lambda
$$

A suitable set of gauge invariants is then provided by

$$
B=Q e^{\Sigma} \quad, \quad \tilde{B}=\tilde{Q} e^{-\Sigma}
$$

These operators play the role of the baryons of the effective "SU(1)" theory below the scale of the $\mathrm{U}(1)$ mass, generated by the $B \wedge F$ coupling in (4.7). Notice for instance that $B \tilde{B}=Q \tilde{Q} \equiv M$ is classically related to the mesonic operator.

Hence, the discussion of the $4 \mathrm{~d}$ instanton amplitudes is most naturally carried out in terms of these variables. Of utmost importance for us is that the structure of operators insertions in (4.6) corresponds to the expansion of $\overline{D B} \overline{D \tilde{B}}$. Thus, the F-term contribution of the instanton at the BPS locus is given by

$$
S_{4 d}=\int d^{4} x d^{2} \theta e^{-T} \overline{D B} \overline{D \tilde{B}}
$$

This contribution is recovered as the $\mathcal{O}\left(\xi^{0}\right)$ term of the instanton amplitude in the near-BPS regime, showing that the full instanton amplitude is in a non-trivial class of the BeasleyWitten cohomology. As in the example in section 3, higher order terms simply correspond to globally defined D-terms, and this can be traced to the fact that the left over zero mode picks up a component along $\bar{\tau}$, which itself picks up a component along $\bar{\theta}$.

As we have argued, switching on a FI forces us to give a vev to $Q$ or $\tilde{Q}$. From the point of view of the induced $4 \mathrm{~d}$ operator, switching on a FI corresponds to interpolating from a situation where the first term in (4.6) dominates (the Beasley-Witten gauge theory analysis) to a situation with a deeply non-BPS instanton where the last term dominates. We study this regime of very non-BPS instantons a bit more in the next section. 
Vevs along the mesonic branch. Before moving on to the non-BPS regime, let us shortly consider another interesting direction in moduli space, namely that in which we do not turn on a vev for $\Sigma$, and move instead along the direction in which $Q$ and $\tilde{Q}$ get the same vev (i.e. the mesonic branch in gauge theory language). In this situation the branes recombine and move together along the plane of the black degeneration, away from the instanton.

In this situation, the instanton and the branes get separated without misaligning the system, and the $\bar{\tau}$ modes on the instanton no longer get lifted. This is perfectly compatible with all of our discussion so far. The setup is similar to the one in section 3.2, with the recombined brane playing the role of the orientifold selecting the preferred $\mathcal{N}=1$ subalgebra. From the same considerations we expect the instanton to generate a $\overline{D \Sigma} \overline{D \Sigma}$ term, which is exactly the term that dominates in (4.6) when both $Q$ and $\tilde{Q}$ get a vev.

\subsection{The deep non-BPS regime}

Let us now describe how the above structure of couplings reproduces the expected physics in the deep non-BPS regime, namely for large $\xi$. Here it is important that motion away from the BPS locus of the instanton forces the recombination of the $4 \mathrm{~d}$ spacefilling Dbranes, namely the scalar components in either $Q$ or $\tilde{Q}$, depending on the sign of $\xi$, acquire a large vev, thereby removing a subset of the instanton zero modes. Consider e.g. the regime where $\tilde{Q}$ acquires a large vev, and the modes $\beta, \tilde{\eta}$ are removed (see eq. (4.1)). So are the bosonic modes $m, \bar{m}$ due to the misalignment of the instanton and the $4 \mathrm{~d}$ D-branes. We are thus left with a U(1) instanton, with the universal sector of zero modes $\theta^{\prime}, \bar{\tau}^{\prime}$, and zero modes $\bar{\beta}, \overline{\tilde{\eta}}$ coupling to the $4 \mathrm{~d}$ chiral multiplet $\bar{Q}$ in the "adjoint" of the recombined $\mathrm{U}(1)$, denoted $\Phi$ in what follows. In the non-BPS regime, i.e. for non-zero BPS phase $\xi$, the universal set can be traded for $\theta, \bar{\theta}$ to yield an instanton amplitude roughly of the form

$$
\int d^{4} x d^{2} \theta d^{2} \bar{\theta} e^{-T^{\prime}} \bar{\Phi}+\text { h.c. }
$$

Here $T^{\prime}$ is the expression of the wrapped volume as a (non-holomorphic) function of the moduli. For instance, in the limit where the instanton looks vertical in the picture of figure 2, the instanton has tension but no (relevant) charge (in a theory with orientifold, the orientifold image would cancel its charge exactly), and $T^{\prime}=\Sigma+\bar{\Sigma}$ where $\Sigma$ controls the vertical position of the white degeneration.

The above is precisely the fermion zero mode content and amplitude expected for a non-BPS U(1) instanton wrapped on a cycle with a chiral intersection with the $4 \mathrm{~d}$ Dbranes. It also matches (4.6) in the limit where we discard the last two terms since they are subleading when the instanton is very non-BPS. In particular, saturating the $\bar{\theta}$ 's with the antichiral pieces in $T^{\prime}$, one can generate amplitudes with two insertions of the $4 \mathrm{~d}$ fermions $\bar{\chi}$ in the $\overline{D \Sigma}$ multiplet, showing the expected behaviour for a (non-isolated) U(1) instanton.

In conclusion, the instanton amplitude satisfies the overall global picture discussed in section 3 , despite the fact that the crucial modes $\bar{\tau}$ have non-trivial interactions. We expect a similar discussion in other examples with interacting $\bar{\tau}$ 's. 
a)

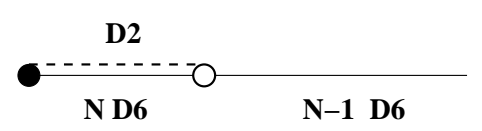

b)

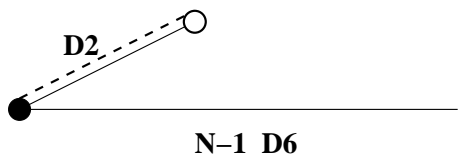

Figure 3: D-brane realization of the $N_{f}=N_{c}-1$ SQCD theory in a geometry of the kind in appendix A. The construction shows that instanton on its BPS locus (a) and away from it (b). The gauge D-branes cannot all recombine and define a non-supersymmetric background for the instanton.

\section{Lifting of fermion zero modes and $4 \mathrm{~d}$ supersymmetry breaking}

In this section we analyze the following possibility. It is in principle possible to consider D-brane instantons which contribute to the superpotential, and which nevertheless can misalign. This would seem to contradict our general statements, based on the counting of goldstinos. However, there is a way out, which automatically comes out in a clever way in the explicit examples below. The modulus that takes the instanton away from the BPS locus simultaneously triggers breaking of $4 \mathrm{~d}$ spacetime supersymmetry. Thus, the instanton actually does not break any exact supersymmetry of the background, and is not forced to have four goldstinos. In certain situations it still has two approximate fermion zero modes (which are not true goldstinos), and the instanton generates $4 \mathrm{~d}$ operators which, in the near supersymmetric regime, can be thought of as an approximate superpotential in the approximately supersymmetric theory, with susy breaking broken by a spacetime D-term.

We discuss two examples. The first corresponds to a D-brane realization of $N_{f}=N_{c}-1$ SQCD, where the instanton generates a superpotential. Motion away from the BPS locus of the D-brane instanton is parameterized by a closed string modulus, which simultaneously induces a $4 \mathrm{~d}$ spacetime D-term potential which cannot be completely canceled and thus breaks $4 \mathrm{~d}$ supersymmetry. The second example corresponds to a U(1) D-instanton with the additional fermion zero modes lifted by closed string fluxes. Again, we find that the modulus taking the instanton away from the BPS locus simultaneously makes the closed string fluxes non-supersymmetric.

\subsection{Gauge theories with non-perturbative superpotential}

Let us consider a D-brane construction, similar to that of previous section, of the $\mathrm{U}\left(N_{c}\right)$ SQCD with $N_{f}=N_{c}-1$ flavours, see figure 3 a. In this case, the D-brane instanton on the BPS locus generates the expected field theory Affleck-Dine-Seiberg superpotential (see e.g. [14 for a detailed computation in the string setup). Let us consider the effect of misaligning the instanton.

In the regime of small misalignment, one can use the gauge theory viewpoint, where it is described as turning on a FI term. Being a D-term, this should not change the $4 \mathrm{~d}$ superpotential. Thus it suggests that a non-BPS instanton induces a superpotential, seemingly in contradiction with our general picture, and with the counting of fermion 
zero modes from goldstinos. There is however no contradiction, as is manifest using the brane picture. In misaligning the instanton by moving the white degeneration up, as in figure $3 \mathrm{~b}$, the gauge D-branes cannot all recombine and one of the color brane misaligns (this is visible in the field theory description as an uncancelled 4d D-term). Thus away from the instanton BPS locus, there is really no supersymmetry in the background, and therefore no need for the instanton to have four goldstinos. Indeed, the modes $\bar{\tau}^{\prime}$ are lifted by couplings with the bosonic zero modes between the instanton and the misaligned color D-brane. The instanton has only two (accidental) zero modes, the $\theta^{\prime}$, and in the near BPS regime it induces operators which can be described in the field theory approximation as a superpotential (in a theory with D-term supersymmetry breaking).

The global description of this kind of example is very different from the previous systems, since in the deep non-BPS regime the background is very non-supersymmetric. We refrain from any further discussion.

Another complementary approach to understand the $N_{f}=N_{c}-1$ SQCD system would be to start with the $N_{f}=N_{c}$ configuration, and add a mass term for one of the flavors. This was done by Beasley and Witten directly in field theory, the result being that the $N_{f}=N_{c}$ instanton is deformed so that the $4 \mathrm{~d}$ operator it leads to has two less fermion insertions. In sketchy terms, we have (at the BPS locus)

$$
\int d^{2} \theta e^{-T} \overline{D Q} \bar{D} \bar{Q}+\int d^{2} \theta M Q \tilde{Q} \longrightarrow \int d^{2} \theta e^{-T}
$$

This is in agreement with the fact that the IR of the $N_{f}=N_{c}$ theory with the mass term should reduce to the $N_{f}=N_{c}-1$ theory. On a similar line, we expect that when we turn on an FI deformation, there will be supersymmetry breaking in the $N_{f}=N_{c}$ theory with massive flavor. This is easy to see in field theory, and also in the brane realization of the theory.

\subsection{Closed string fluxes}

A well-known mechanism to lift zero modes is to consider D-brane instantons in the presence of $\mathcal{N}=1$ closed string background fluxes [40, 41, 33]. As the fluxes break the Calabi-Yau $\mathcal{N}=2$ supersymmetry down to $\mathcal{N}=1$ on top of instanton worldvolume, there is a priori no reason for the additional zero modes $\bar{\tau}$ of section 3 to be present. Hence one would expect that, in the presence of fluxes, one can obtain U(1) instantons that contribute to the nonperturbative superpotential. Since in principle such instantons can misalign, this would lead to contradiction with general arguments of counting of goldstinos. In the following we would like to argue, using the viewpoint of the world-volume fermionic action, that this is not the case.

The Dirac action for the fermionic modes on a $\mathrm{D} p$-instanton reads 34

$$
\Theta P_{\kappa}^{\mathrm{Dp}} \mathcal{D} \Theta,=\Theta P_{\kappa}^{\mathrm{Dp}}\left(\mathcal{M}^{m n} \Gamma_{m} \mathcal{D}_{n}-\frac{1}{2} \mathcal{O}\right) \Theta
$$

where $\Theta$ are the fermionic superembedding variables, $P_{\kappa}^{\mathrm{Dp}}$ is the $\kappa$-symmetry projector, which for an instanton with BPS phase $\xi$ selects the zero modes of the form (3.10), and $\mathcal{D}_{n}$ and $\mathcal{O}$ are the operators appearing in the gravitino and dilatino variations, pull-backed to 
the D-instanton worldvolume. Finally, $\mathcal{M}^{m n}$ is a matrix which depends on the instanton worldvolume flux $\mathcal{F}$, and which for $\mathcal{F}=0$ reduces to $g^{m n}$.

From (5.2) one can see that a instanton fermionic zero mode needs to satisfy two requirements. First, in order to be a true fermion mode (rather than a $\kappa$-symmetry parameter) it must not be projected out by $P_{\kappa}^{\text {Dp }}$; and, second, it must be annihilated by the operator $\mathcal{D}$. Let us see how these requirements apply for the $\mathrm{U}(1)$ instanton of section 3 , which we will again assume to be rigid and isolated. Hence, the space of zero mode candidates is contained in table 1. It is important that, since the instanton is not mapped to itself under the orientifold, one can use (5.2) on the covering space.

If our background is Calabi-Yau, it is clear that any linear combination of the zero modes in table 1 is annihilated by $\mathcal{D}$, for they are all individually annihilated by $\mathcal{D}_{n}$ and $\mathcal{O}$. Hence, the rotated modes (3.10) selected by $P_{\kappa}^{\mathrm{Dp}}$ are automatically zero modes, whether we are in the BPS locus $(\xi=0$ or $\pi$ ) or away from it, and we recover the well-known result that such $\mathrm{U}(1)$ instanton has four zero modes.

If we now turn on background fluxes the operators $\mathcal{D}_{n}$ and $\mathcal{O}$ get deformed, and it is no longer true that $\mathcal{D}_{n} \tau=\mathcal{D}_{n} \bar{\tau}=\mathcal{O} \tau=\mathcal{O} \bar{\tau}=0$. However, we can use the fact that for $\xi \neq 0$, the instanton is non-BPS and thus has four zero modes, which are the four goldstinos of the broken $\mathcal{N}=1$ supersymmetry, namely $\mathcal{D} \bar{\tau}^{\prime}=0$ and $\mathcal{D} \theta^{\prime}=0$, to yield

$$
0=\mathcal{D} \bar{\tau}^{\prime}=\cos (\xi / 2) \mathcal{D} \bar{\tau}+\sin (\xi / 2) \mathcal{D} \bar{\theta}=\cos (\xi / 2) \mathcal{D} \bar{\tau}=0,
$$

and similarly for $\theta^{\prime}$. Here we have used that $\xi$ is constant and that $\mathcal{D}_{n} \bar{\theta}=\mathcal{O} \bar{\theta}=0$, for this mode comes from the background Killing spinor.

We thus find that, anywhere close to the BPS locus $\xi=0, \bar{\tau}$ is annihilated by $\mathcal{D}$. By continuity of the spectrum of such differential operator, we conclude that it must also be so for $\xi=0$. Thus, it is not possible to turn on this kind of closed string backgrounds to lift the additional fermion zero modes $\bar{\tau}$.

Notice that the argument above does not apply if the instanton cannot misalign via a $\xi \neq 0$. This includes the case of instantons invariant under some orientifold action, of gauge group either $O(1)$ or $\operatorname{USp}(2)$, that were considered in 441] to find new contributions to the superpotential. ${ }^{9}$

Notice that there is a further way out of the argument, involving breaking of $4 \mathrm{~d}$ supersymmetry in a way similar to section 5.1. There may exist flux background which lift the additional fermion zero modes, and allow the instanton to contribute to the superpotential, if the flux background is non-supersymmetric for non-zero $\xi$. Indeed, in that case taking $\xi \neq 0$ would not only mean that the instanton is non-BPS, but also that the closed string background is $\mathcal{N}=0$. In that case (5.3) need not be true because, away from $\xi=0$, $\mathcal{D}_{n} \bar{\theta}=\mathcal{O} \bar{\theta}=0$ no longer holds. In addition, we cannot claim that there is a minimum of four fermion zero modes coming from goldstinos, for there are no bulk supersymmetries to be broken.

To our knowledge, lifting of fermion zero modes by the latter kind of fluxes has not been much considered in the literature. As a sketchy example, along the lines of section 5.3

\footnotetext{
${ }^{9}$ In addition, as pointed out in [41], in this case the fermionic action (5.2) is not valid, and needs to be further projected by the orientifold action.
} 
of [33], we may consider a toroidal orientifold compactification ${ }^{10}$ with $J=\sum d z_{i} d \bar{z}_{i}$ and a supersymmetric primitive $(2,1) 3$-form flux $G_{\overline{1} 23}$. Consider a D3-brane instanton wrapped on $z_{1}, z_{2}$ and magnetized with a world-volume primitive flux $F_{1 \overline{2}}$, so that it is BPS. As argued in [33] this flux could remove the additional fermion zero modes in the universal sector $\bar{\tau}$. This would seem to contradict our general arguments, since the BPS phase of the D3-brane can be misaligned by turning on a component of the Kahler form along $d \bar{z}_{1} d z_{2}+d z_{1} d \bar{z}_{2}$, so that the world-volume flux is no longer primitive and the instanton becomes non-BPS. However, this simultaneously makes the 3-form flux non-primitive, and thus breaks spacetime supersymmetry.

For the most studied setup of type IIB with 3-form fluxes on warped genuine CalabiYau geometries [42, 43], the 3-form flux primitivity condition is automatic and the fluxes do not affect the (Kähler) moduli controlling the FI parameter $\xi$. Hence, by our discussion above, they will never succeed in lifting the zero modes $\bar{\tau}$ unless they break supersymmetry at the same time. This was partially observed for IIB D3-brane instantons in 3-form flux backgrounds in [33], but should hold in full generality. Note that the above analysis provides a new, deeper understanding of the negative results in [40, 41, 33].

\subsection{Effects of additional instantons}

A further possibility is to consider the lifting or absorption of additional fermion zero modes of an instanton by another. Refraining from a general analysis, let us briefly sketch an example of the interplay between lifting of additional zero modes and BPS misalignment of $\mathrm{U}(1)$ instanton.

In 28] a mechanism to saturate the extra fermion zero modes of a U(1) instanton was presented. Namely, a U(1) instanton in the presence of an additional $O(1)$ instanton can produce a two-instanton effect which contributes to the superpotential. In a precise sense, the $O(1)$ instanton produces a non-perturbative lifting of the additional zero modes of the $\mathrm{U}(1)$ instanton. This would seem to contradict our general argument above, since there is a parameter which can misalign the $\mathrm{U}(1)$ instanton and make it non-BPS. The resolution of the puzzle is that the misalignment is realized when only the $\mathrm{U}(1)$ instanton is present; in the presence of the $O(1)$ instanton, namely precisely when the additional zero modes are lifted, the same parameter actually turns the two-instanton system into a BPS oneinstanton system. Namely, there is line of marginal stability for the U(1) instanton (with four fermion zero modes), which is just a line of threshold stability for the U(1)-O(1) twoinstanton system (with two fermion zero modes). Thus no contradiction with our general picture is found.

This provides an amusing example of a behaviour complementary to the previous systems. In this case, the mechanism that provides the lifting of extra zero modes does not break the $4 \mathrm{~d}$ supersymmetry, but rather restores the BPS property for the new multiinstanton system.

\footnotetext{
${ }^{10}$ For simplicity we ignore tadpole cancellation, or include suitable antibranes away from the system of interest so that they do not modify the argument.
} 
It is easy to device other systems of several instantons and show that such systems manage in clever ways to always comply as expected with the general rules of counting of goldstinos. Other examples following a similar pattern have appeared in [25].

\section{Conclusions}

In this paper we have completed the picture of instanton amplitudes as they cross lines of BPS stability of different kinds. BPS instantons contributing to the superpotential have at worst lines of threshold stability, where they split into mutually BPS instantons, which reconstruct the non-perturbative superpotential. BPS instantons contributing to higher F-terms can also have lines of marginal stability, beyond which they turn into (possibly multi-instanton) non-BPS systems, which reproduce the same F-term (rewritten locally as a D-term), modulo globally defined D-terms.

The picture is consistent, in a non-trivial way, with standard wisdom of instanton fermion zero mode counting, and with holomorphy of $4 \mathrm{~d} \mathcal{N}=1$ F-terms. An important lesson in this story has been the role of the Beasley-Witten cohomological structure of higher F-terms. Although the structure of this cohomology is unfamiliar and quantitative statements are hard to make explicit, the instanton zero mode structure automatically reproduces the appropriate features. It would be interesting to develop a formalization of these results, beyond the examples we have provided.

It would also be interesting to continue understanding the behaviour of instanton amplitudes globally in moduli space. In $\mathcal{N}=2$ language we have focused on hypermultiplet moduli space (complex structure moduli space for IIA, Kahler for IIB). It would be interesting to also explore further the dependence on vector multiplet moduli space (Kahler for IIA, complex for IIB), which essentially control the 1-loop prefactors of the exponential term in the instanton amplitude. These moduli are naturally related to F-terms on the world-volume of the instanton, and therefore the holomorphic dependence on the moduli is quite straightforward. Still one may expect interesting lessons also from a deeper look into this dependence, and we hope that some of the concepts we discussed in this work will be useful in this new setup.

For instance, it is easy to consider systems of BPS systems which can become nonBPS due to an uncancelled world-volume F-term. Possibly the simplest system of this kind is given by a D1-brane instanton wrapped on the non-trivial two-cycle of a two-center hyperkahler ALE geometry. There is a triplet of blow-up parameters, which, with respect to some preferred $4 \mathrm{~d} \mathcal{N}=1$ supersymmetry, couple to the instanton as a real worldvolume FI term, and a holomorphic world-volume F-term (a superpotential linear in a complex bosonic zero mode). As in our examples of D-term misalignment in this paper, the F-term misaligned instanton has four goldstinos and generates a $4 \mathrm{~d}$ non-perturbative operator, which is writable as a D-term locally in moduli space and which reduces to a $4 \mathrm{~d}$ non-perturbative higher F-term as one moves the BPS locus. In this case, the picture of F-term misalignment is identical to D-term misalignment since the underlying hyperkahler geometry implies a tri-holomorphic symmetry relating the different components in the triplet of blowing-up parameters. We nevertheless expect a similar behaviour for F-term 
misalignment in more generic situations, since the basic facts relate to general properties of counting of goldstinos and properties of higher F-terms.

We expect interesting forthcoming fundamental results and interesting applications from continuing the analysis of instanton amplitudes globally in moduli space.

\section{Acknowledgments}

We thank L. Ibáñez, T. Weigand for useful discussions. A.M.U. thanks M. González for encouragement and support. I.G.-E. thanks the CERN Theory division for hospitality, and N. Hasegawa for kind support. This work has been supported by the European Commission under RTN European Programs MRTN-CT-2004-503369, MRTN-CT-2004-005105, by the CICYT (Spain) and the Comunidad de Madrid under project HEPHACOS P-ESP-00346. The work of I.G.-E. was financed by the Gobierno Vasco PhD fellowship program.

\section{A. Some useful geometries}

Here we describe a set of geometries, introduced in [44] and already used in [28] in a similar context, and which we use in several of our explicit examples. They are noncompact geometries, but they suffice to provide instanton effects and transitions as long as they involve just the local structure of compact cycles.

Consider the class of local Calabi-Yau manifolds, described by

$$
\begin{array}{r}
x y=\prod_{k=1}^{P}\left(z-a_{k}\right) \\
x^{\prime} y^{\prime}=\prod_{k^{\prime}=1}^{P^{\prime}}\left(z-b_{k}^{\prime}\right)
\end{array}
$$

It describes two $\mathbf{C}^{*}$ fibrations, parameterized by $x, y$ and $x^{\prime}, y^{\prime}$, varying over the complex plane $z$, and degenerating at the locations $a_{i}, b_{i}$ respectively. The local geometry contains lagrangian 3-cycles obtained by fibering the two $\mathbf{S}^{\mathbf{1}}$ 's in the two $\mathbf{C}^{*}$ fibers over segments joining pairs of degeneration points on the base. Segments joining pairs of $a$-type degenerations or pairs of $b$-type degenerations lead to 3 -cycles with topology $\mathbf{S}^{\mathbf{2}} \times \mathbf{S}^{\mathbf{1}}$, while segments joining $a$ - and $b$-type degenerations lead to 3 -cycles with topology $\mathbf{S}^{\mathbf{3}}$. We denote $\left[p_{1}, p_{2}\right]$ the 3 -cycle associated to the pair of degeneration points $p_{1}, p_{2}$.

Introducing the holomorphic 3 -form

$$
\Omega=\frac{d x}{x} \frac{d x^{\prime}}{x^{\prime}} d z
$$

the 3 -cycle $\left[p_{1}, p_{2}\right]$ is calibrated by the form $e^{i \theta} \Omega$, where $\theta$ is the angle of the segment $\left[p_{1}, p_{2}\right]$ with the real axis in the $z$-plane. Namely $\left.\operatorname{Im}\left(e^{i \theta} \Omega\right)\right|_{\left[p_{1}, p_{2}\right]}=0$, where $\left.\right|_{\left[p_{1}, p_{2}\right]}$ denotes restriction to the 3-cycle. Segments parallel in the $z$-plane define 3-cycles preserving a common supersymmetry. Our configurations will be $\mathrm{d} \mathcal{N}=1$ supersymmetric, with the preferred supersymmetry associated to segments parallel to the real axis in $z$. 
We will consider stacks of 4d spacefilling D6-branes and/or euclidean D2-branes wrapping the different 3-cycles, and describe the non-perturbative superpotentials arising from these configurations. The open string modes and their interactions are easy to determine. For instance, each stack of $N$ D6-branes on a 3-cycle leads to a $\mathrm{U}(N)$ gauge group in a vector multiplet of $\mathcal{N}=1$ supersymmetry for 3 -cycles of $\mathbf{S}^{\mathbf{3}}$ topology, and of $\mathcal{N}=2$ supersymmetry for 3-cycles of $\mathbf{S}^{\mathbf{2}} \times \mathbf{S}^{\mathbf{1}}$ topology. The angle $\theta$ introduced above determines the precise supersymmetry preserved by the corresponding set of branes. Also, two D6-branes wrapping two 3-cycles involving one common degeneration point lead to a vector-like pair of bi-fundamental chiral multiplets, arising from open strings in the intersection of 3-cycles (which is topologically $\mathbf{S}^{\mathbf{1}}$, coming from the $\mathbf{C}^{*}$ that does not degenerate at the intersection).

As discussed in [44] one can perform T-dualities along the two $\mathbf{S}^{\mathbf{1}}$ directions, and map the configuration to a Hanany-Witten setup of $P$ NS-branes (along 012345) and $P^{\prime}$ NS'branes (along 012389), with D4-branes (along 01236) suspended among them, in a flat space geometry with a periodic coordinate $x^{6}$. The gauge theory content described above follows from the standard rules in this setup (see [45]). This picture also facilitates the computation of the superpotential, whose general discussion we skip, but which we present in our concrete example below.

\section{References}

[1] K. Becker, M. Becker and A. Strominger, Five-branes, membranes and nonperturbative string theory, Nucl. Phys. B 456 (1995) 130 hep-th/9507158.

[2] E. Witten, Non-perturbative superpotentials in string theory, Nucl. Phys. B 474 (1996) 343 hep-th/9604030.

[3] J.A. Harvey and G.W. Moore, Superpotentials and membrane instantons, hep-th/9907026.

[4] E. Witten, World-sheet corrections via D-instantons, JHEP 02 (2000) 030 hep-th/9907041.

[5] S. Kachru, R. Kallosh, A. Linde and S.P. Trivedi, De Sitter vacua in string theory, Phys. Rev. D 68 (2003) 046005 hep-th/0301240.

[6] F. Denef, M.R. Douglas and B. Florea, Building a better racetrack, JHEP 06 (2004) 034 hep-th/0404257.

[7] F. Denef, M.R. Douglas, B. Florea, A. Grassi and S. Kachru, Fixing all moduli in a simple F-theory compactification, Adv. Theor. Math. Phys. 9 (2005) 861 hep-th/0503124.

[8] R. Blumenhagen, M. Cvetič and T. Weigand, Spacetime instanton corrections in $4 D$ string vacua - the seesaw mechanism for D-brane models, Nucl. Phys. B 771 (2007) 113 hep-th/0609191.

[9] L.E. Ibáñez and A.M. Uranga, Neutrino Majorana masses from string theory instanton effects, JHEP 03 (2007) 052 hep-th/0609213.

[10] M. Haack, D. Krefl, D. Lüst, A. Van Proeyen and M. Zagermann, Gaugino condensates and D-terms from D7-branes, JHEP 01 (2007) 078 hep-th/0609211.

[11] B. Florea, S. Kachru, J. McGreevy and N. Saulina, Stringy instantons and quiver gauge theories, JHEP 05 (2007) 024 hep-th/0610003. 
[12] L.E. Ibáñez, A.N. Schellekens and A.M. Uranga, Instanton induced neutrino Majorana masses in CFT orientifolds with MSSM-like spectra, JHEP 06 (2007) 011 arXiv:0704.1079.

[13] M. Billó et al., Classical gauge instantons from open strings, JHEP 02 (2003) 045 hep-th/0211250.

[14] N. Akerblom, R. Blumenhagen, D. Lüst, E. Plauschinn and M. Schmidt-Sommerfeld, Non-perturbative SQCD superpotentials from string instantons, JHEP 04 (2007) 076 hep-th/0612132.

[15] M. Bianchi and E. Kiritsis, Non-perturbative and flux superpotentials for type I strings on the $Z_{3}$ orbifold, Nucl. Phys. B 782 (2007) 26 hep-th/0702015.

[16] R. Argurio, M. Bertolini, G. Ferretti, A. Lerda and C. Petersson, Stringy instantons at orbifold singularities, JHEP 06 (2007) 067 arXiv:0704.0262].

[17] M. Bianchi, F. Fucito and J.F. Morales, D-brane instantons on the $T^{6} / Z_{3}$ orientifold, JHEP 07 (2007) 038 arXiv:0704.0784.

[18] R. Argurio, M. Bertolini, S. Franco and S. Kachru, Metastable vacua and D-branes at the conifold, JHEP 06 (2007) 017 hep-th/0703236.

[19] O. Aharony, S. Kachru and E. Silverstein, Simple stringy dynamical SUSY breaking, Phys. Rev. D 76 (2007) 126009 arXiv:0708.0493.

[20] M. Aganagic, C. Beem and S. Kachru, Geometric transitions and dynamical SUSY breaking, Nucl. Phys. B 796 (2008) 1 arXiv:0709.4277.

[21] M. Billó et al., Instanton effects in $N=1$ brane models and the Kähler metric of twisted matter, JHEP 12 (2007) 051 arXiv:0709.0245.

[22] N. Akerblom, R. Blumenhagen, D. Lüst and M. Schmidt-Sommerfeld, Instantons and holomorphic couplings in intersecting D-brane models, JHEP 08 (2007) 044 arXiv:0705.2366.

[23] M. Bianchi and J.F. Morales, Unoriented D-brane instantons vs heterotic worldsheet instantons, JHEP 02 (2008) 073 arXiv:0712.1895.

[24] R. Blumenhagen and M. Schmidt-Sommerfeld, Power towers of string instantons for $N=1$ vacua, arXiv:0803.1562.

[25] M. Cvetič, R. Richter and T. Weigand, (Non-)BPS bound states and D-brane instantons, arXiv:0803.2513.

[26] Y. Matsuo, J. Park, C. Ryou and M. Yamamoto, D-instanton derivation of multi-fermion F-terms in supersymmetric $Q C D$, arXiv:0803.0798.

[27] F. Denef, (Dis)assembling special Lagrangians, hep-th/0107152.

[28] I. Garcia-Etxebarria and A.M. Uranga, Non-perturbative superpotentials across lines of marginal stability, JHEP 01 (2008) 033 arXiv:0711.1430].

[29] J. de Boer, F. Denef, S. El-Showk, I. Messamah and D. Van den Bleeken, Black hole bound states in $A d S_{3} \times S^{2}$, arXiv:0802.2257.

[30] L.E. Ibáñez and A.M. Uranga, Instanton induced open string superpotentials and branes at singularities, JHEP 02 (2008) 103 arXiv:0711.1316. 
[31] C. Beasley and E. Witten, New instanton effects in supersymmetric QCD, JHEP 01 (2005) 056 hep-th/0409149.

[32] C. Beasley and E. Witten, New instanton effects in string theory, JHEP 02 (2006) 060 hep-th/0512039.

[33] R. Blumenhagen, M. Cvetič, R. Richter and T. Weigand, Lifting D-instanton zero modes by recombination and background fluxes, JHEP 10 (2007) 098 arXiv:0708.0403.

[34] L. Martucci, J. Rosseel, D. Van den Bleeken and A. Van Proeyen, Dirac actions for D-branes on backgrounds with fluxes, Class. and Quant. Grav. 22 (2005) 2745 hep-th/0504041.

[35] M. Berkooz, M.R. Douglas and R.G. Leigh, Branes intersecting at angles, Nucl. Phys. B 480 (1996) 265 hep-th/9606139.

[36] A. Hanany and A. Iqbal, Quiver theories from D6-branes via mirror symmetry, JHEP 04 (2002) 009 hep-th/0108137.

[37] B. Feng, Y.-H. He, K.D. Kennaway and C. Vafa, Dimer models from mirror symmetry and quivering amoebae, hep-th/0511287.

[38] C. Petersson, Superpotentials from stringy instantons without orientifolds, JHEP 05 (2008) 078 arXiv:0711.1837.

[39] M. Dine, N. Seiberg and E. Witten, Fayet-Iliopoulos terms in string theory, Nucl. Phys. B 289 (1987) 589.

[40] P.K. Tripathy and S.P. Trivedi, D3 brane action and fermion zero modes in presence of background flux, JHEP 06 (2005) 066 hep-th/0503072.

[41] E. Bergshoeff, R. Kallosh, A.-K. Kashani-Poor, D. Sorokin and A. Tomasiello, An index for the Dirac operator on D3 branes with background fluxes, JHEP 10 (2005) 102 hep-th/0507069.

[42] K. Dasgupta, G. Rajesh and S. Sethi, M theory, orientifolds and G-flux, JHEP 08 (1999) 023 hep-th/9908088.

[43] S.B. Giddings, S. Kachru and J. Polchinski, Hierarchies from fluxes in string compactifications, Phys. Rev. D 66 (2002) 106006 hep-th/0105097.

[44] H. Ooguri and C. Vafa, Geometry of $N=1$ dualities in four dimensions, Nucl. Phys. B 500 (1997) 62 hep-th/9702180.

[45] A. Giveon and D. Kutasov, Brane dynamics and gauge theory, Rev. Mod. Phys. 71 (1999) 983 hep-th/9802067. 